\title{
Mesialização de molar por meio de mini-implantes: uma solução para perda de dentes com comprometimento radicular
}

\section{Molar mesialization by means of mini-implants: a solution for tooth loss with root involvement}

\section{Mesialización molar mediante mini-implantes: una solución para la pérdida de dientes con afectación radicular}

\author{
Andréia Regina Boff Lemos ${ }^{1}$ \\ Márcio Feitosa Borges ${ }^{2}$ \\ Renan Morais Peloso \\ Karina Maria Salvatore Freitas iD \\ Fabrício Pinelli Valarelli \\ 1 andreiabrlemos@hotmail.com \\ 2 marciofeitosaborges@gmail.com \\ Endereço para correspondência: \\ Karina Maria Salvatore Freitas \\ Centro Universitário Ingá - UNINGÁ \\ Rodovia PR 317, 6114 \\ 87035-510 - Maringá - Paraná - Brasil \\ E-mail:kmsf@uol.com.br
}

Recebidlo: 26.08.2020

Aceito: 08.09.2020

RESUMO

A utilização de mini-implantes para a mesialização de molares na rotina da clínica ortodôntica tem produzido resultados bastante satisfatórios, visto que tal movimentação ocorre com maior controle do profissional evitando-se os efeitos colaterais indesejáveis nos dentes adjacentes, pois toda a força aplicada é realizada no dispositivo de ancoragem fixa. Este trabalho tem o intuito de relatar o tratamento de uma paciente adulta com a indicação clínica de extração de um molar permanente, cujo espaço da extração foi fechado por meio de mecânica de mesialização apoiada em mini-implante. Ao final do tratamento, obteve-se o fechamento total do espaço proporcionando uma oclusão satisfatória.

PALAVRAS-CHAVE: Procedimentos de ancoragem ortodôntica. Ortodontia. Implantes dentários.

\section{ABSTRACT}

The use of mini-implants for molar mesialization in the routine of orthodontic clinics has produced quite satisfactory results, since such movement occurs with greater control by the professional, avoiding undesirable side effects on adjacent teeth, as all the applied force is performed on the fixed anchorage device. This work aims to report the treatment of an adult patient with the clinical indication of extraction of a permanent molar, whose extraction space was closed by means of mesialization mechanics 
supported by a mini-implant. At the end of treatment, it was obtained the total closure of the space providing a satisfactory occlusion.

KEYWORDS: Orthodontic anchorage procedures. Orthodontics. Dental implants.

\section{RESUMEN}

El uso de mini-implantes para mesialización de molares en la rutina de la clínica de ortodoncia ha producido resultados bastante satisfactorios, ya que dicho movimiento se produce con mayor control por parte del profesional, evitando efectos secundarios indeseables en los dientes adyacentes, ya que se realiza toda la fuerza aplicada. en el dispositivo de anclaje fijo. Este trabajo tiene como objetivo reportar el tratamiento de un paciente adulto con la indicación clínica de extracción de un molar permanente, cuyo espacio de extracción se cerró mediante mecánicas de mesialización sostenidas por un mini-implante. Al final del tratamiento, el espacio se cerró por completo, proporcionando una oclusión satisfactoria.

PALABRAS CLAVE: Métodos de anclaje en ortodoncia. Ortodoncia. Implantes dentales. 


\section{INTRODUÇÃO}

Na rotina da clínica de ortodontia em adultos, tem sido alta a procura por tratamentos que possam resolver o problema de perdas dentárias, sem comprometer a estética do paciente. É comum a prática de fechamento de espaços devido à perda de dentes permanentes, porém tal movimento para mesial dos molares pode levar a movimentações dentárias indesejáveis do segmento anterior do arco se a ancoragem não for suficiente' .

Métodos tradicionais de ancoragem intra e extra-orais são utilizados até hoje, como botão de Nance, barra palatina, arco lingual, $\mathrm{AEB}$, entre outros, mas todos possuem suas indicações e limitações ${ }^{2-3}$. Os mini-implantes aparecem como mais uma opção para ancoragem esquelética ${ }^{4}$.

A utilização de mini-implantes na clínica ortodôntica tem sido utilizada rotineiramente. Dentre estes protocolos para tratar ausências ou perdas dentárias, a mesialização de molares vem sendo utilizada com grande frequência, visto que, a instalação do mini-implante para a realização da mecânica, além do baixo custo é realizada através de um procedimento simples, rápido e, na maioria das vezes, indolor ${ }^{5-6}$.

A mesialização de molares realizada por meio de ancoragem esquelética consiste no movimento mesial dos dentes sem que haja reações nos segmentos anteriores do arcol, como pode ocorrer quando isso é realizado sem a utilização de ancoragem esquelética ${ }^{7}$. Tal movimentação está indicada para fechamentos de espaço causados devido a perdas dentárias de permanentes, fechamento de espaços devido a agenesias e, extrações de dentes permanentes com prognóstico duvidoso (dentes com problemas endodônticos, lesões de furca, infiltrações em próteses, perdas ósseas verticais ou horizontais) ${ }^{1}$.

Com a utilização da mecânica de mesialização com mini-implantes, o tratamento ortodôntico acontece de forma simplificada e com os efeitos colaterais indesejados minimizados, proporcionando por meio de uma ancoragem absoluta uma oclusão favorável e uma estética adequada ${ }^{2}$.

Este artigo tem o intuito de demonstrar um tratamento de uma paciente adulta com a indicação clínica de extração de um molar permanente, cujo espaço da extração foi fechado por meio de mecânica de mesialização apoiada em mini-implante, onde ao final do tratamento obteve-se o fechamento total do espaço proporcionando uma oclusão satisfatória.

\section{RELATO DE CASO}

Paciente D.B.F., 23 anos, procurou a clínica de ortodontia com a queixa principal de presença de diastemas no arco superior. $\mathrm{Na}$ análise extrabucal observou-se um perfil convexo, padrão mesofacial, e harmonia entre os terços faciais (Figura 1 A-C).

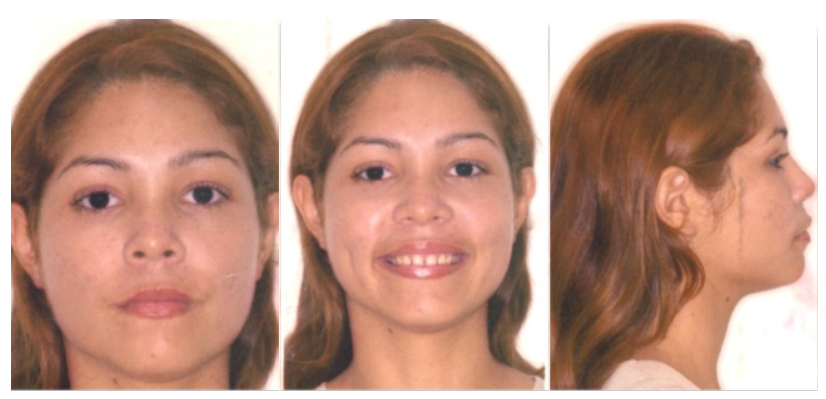

Figura 1 A-C - Fotografias extrabucais iniciais.

$\mathrm{Na}$ análise intrabucal pode-se observar uma máoclusão de Classe I bilateral com mordida profunda e presença de diastemas na região anterior do arco superior. No arco inferior observa-se uma restauração extensa no dente 46 (Figura 2A-E).

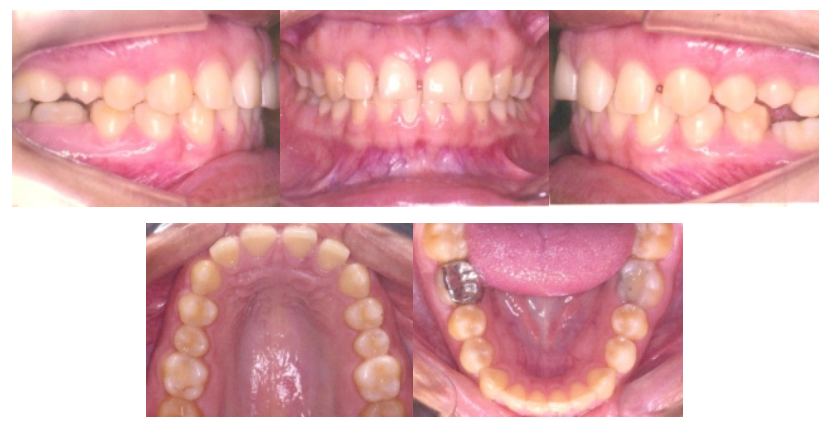

Figura 2 A-E - Fotografias intrabucais iniciais.

Na radiografia panorâmica inicial observa-se presença dos terceiros molares, lesão periapical no dente 46 (Figura 3).

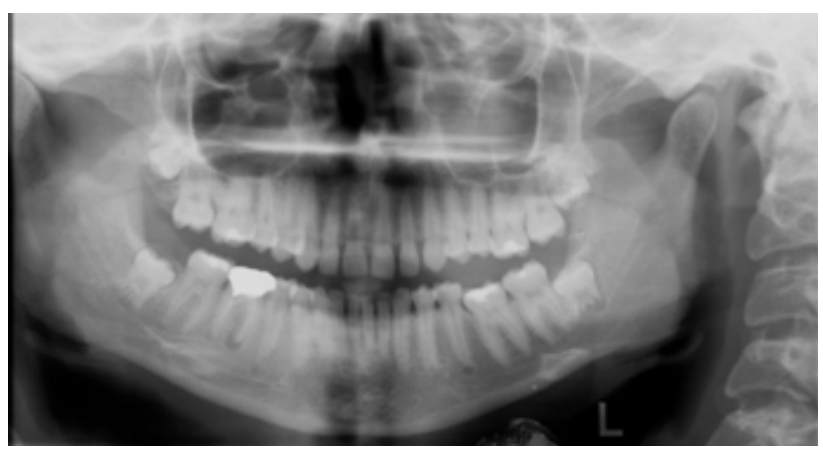

Figura 3 - Radiografia panorâmica inicial.

Para melhor diagnóstico da lesão do dente 46, observou-se a lesão periapical e a presença de um leve espessamento na região de furca do dente, sendo possível fechar o diagnóstico de extração indicada (Figura 4). 


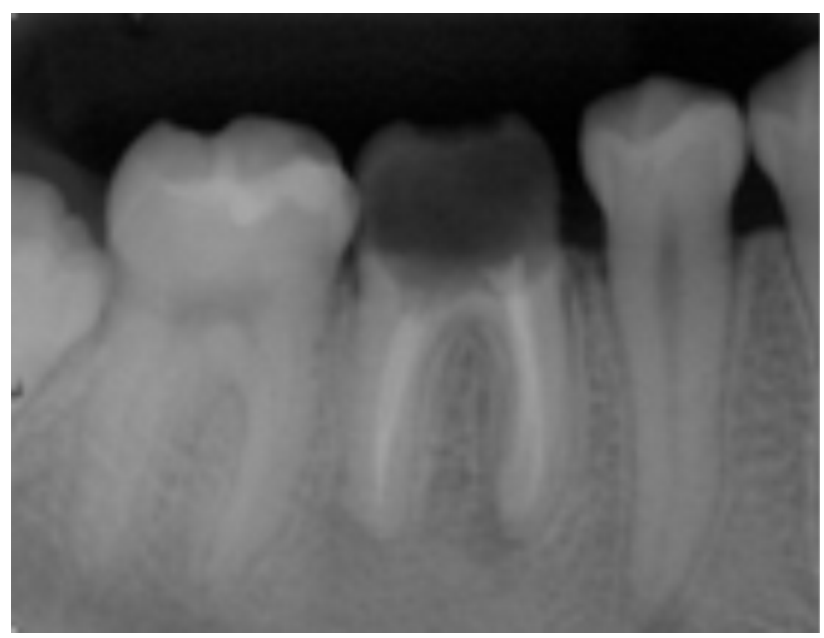

Figura 4 - Radiografia periapical do dente 46.

Na telerradiografia observou-se o perfil convexo, e a normalidade das estruturas adjacentes (Figura 5).

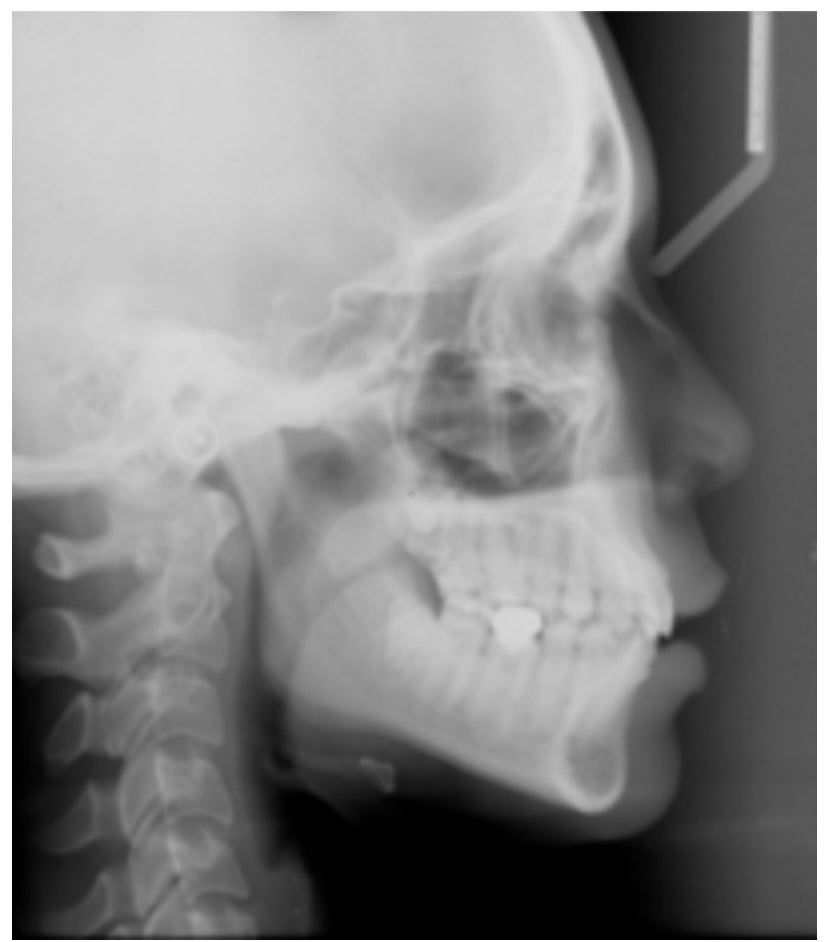

Figura 5 - Telerradiografia inicial.

\section{Plano de Tratamento}

Diante das evidências clínicas e radiográficas, optou-se pela extração do dente 46 , mesialização do dente 47 com mini-implante até a erupção do dente 48 para posteriormente ser mesializado.

\section{Tratamento}

Após a colagem dos acessórios superiores foi utilizado arcos com acentuação de curva de Spee para posterior colagem dos acessórios inferiores, visto que não havia espaço suficiente para que a colagem fosse feita devido a sobremordida anterior. Pode-se notar o aumento dos diastemas localizados na região anterossuperior e a presença de overbite de $-1 \mathrm{~mm}$ na região dos incisivos centrais (Figura 6 A-E).

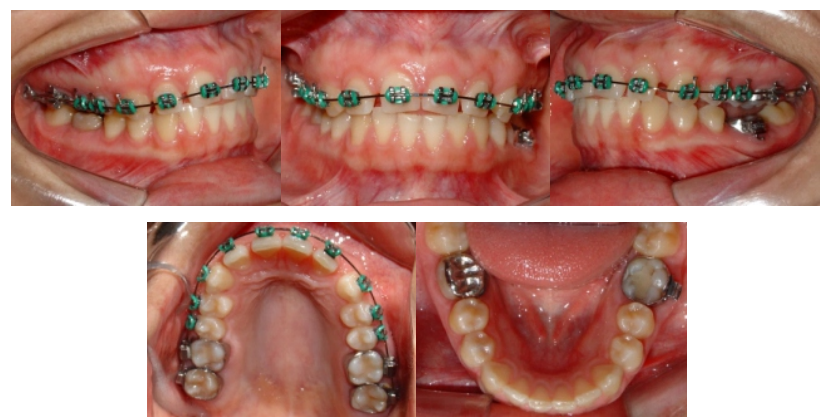

Figura 6 A-E - Fotografias após a acentuação da curva de Spee favorecendo a colagem inferior.

Após a extração do dente 46, o alinhamento e nivelamento fez-se por meio de arcos redondos de NiTi 0.014", 0.016", 0.018 ". A acentuação e reversão de curva de Spee foi realizada por meio de arcos redondos de aço 0.018" e 0.020". Após serem utilizados arcos retangulares de NiTi 0.019" X 0.025", fez-se o uso de arcos retangulares de aço 0.019" X 0.025", momento este que se iniciou o fechamento dos espaços superiores por meio de elásticos em cadeia e instalou-se o mini-implante entre os dentes 44 e 45, iniciando-se a mecânica de mesialização com cantilever ligado com elástico corrente do dente 47 ao mini-implante (Figura 7 A-E e 8).

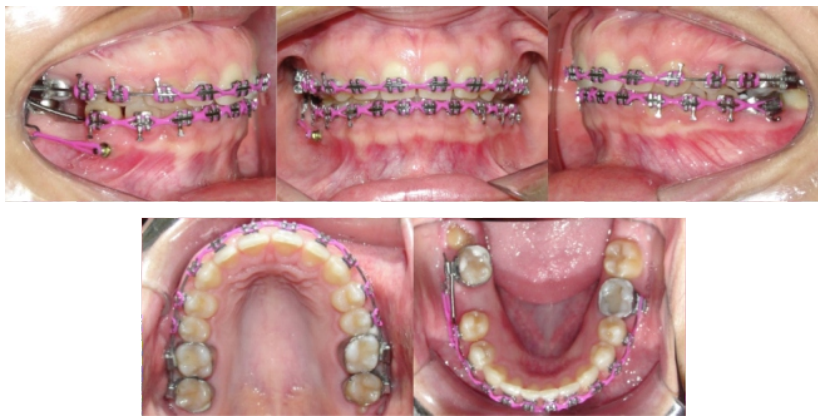

Figura 7A-E - Fotografia do início da mesialização do 47.

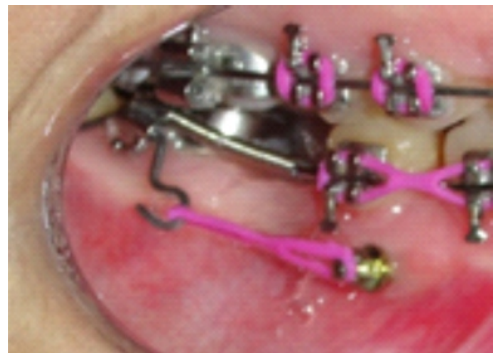

Figura 8 - Fotografia do cantilever com a utilização do elástico em cadeia para a mesialização. 


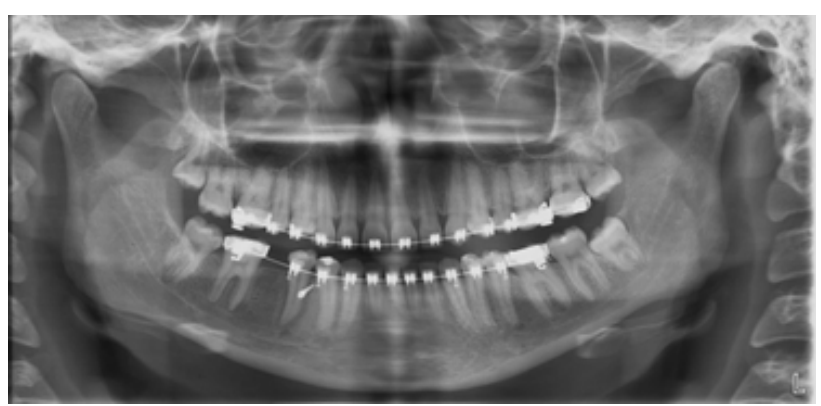

Figura 9 - Panorâmica durante o tratamento de mesialização.

A finalização do fechamento dos espaços se deu pelo uso de elástico em cadeia, concomitante ao uso de elásticos de Classe II bilateral (Figura 10 A-E).
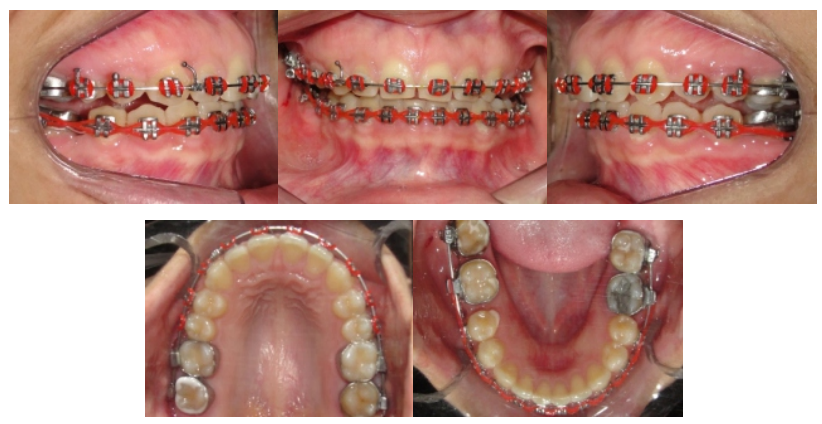

Figura 10 A-E - Fotografias do fechamento dos espaços.

Após o fechamento dos espaços foi removido o aparelho ortodôntico e instalado placa de Hawley superior e $3 \times 3$ inferior (Figura $11 \mathrm{~A}-\mathrm{H}$ ).

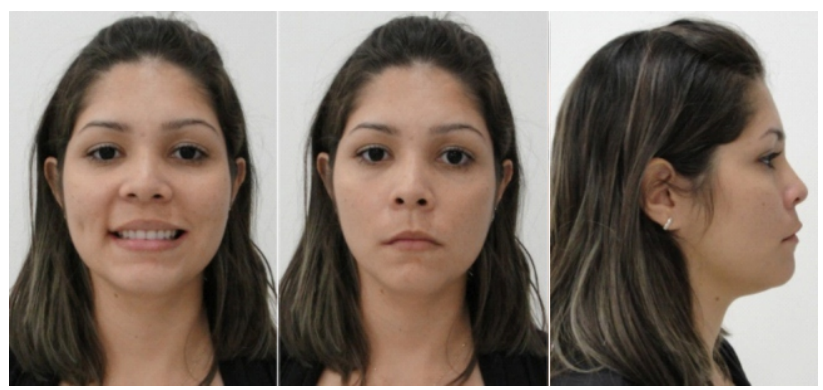

Figura 11 A-C - Fotografias finais extrabucais.
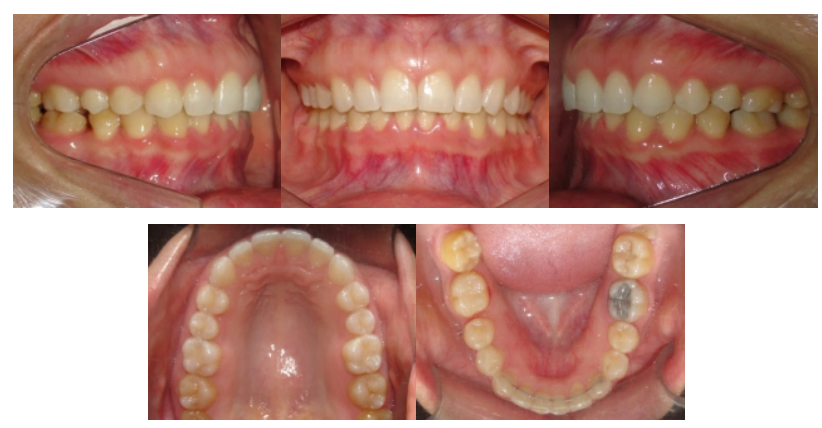

Figura 12 A-E - Fotografias intrabucais finais.
$\mathrm{Na}$ radiografia panorâmica final observa-se o paralelismo das raízes (Figura 13).

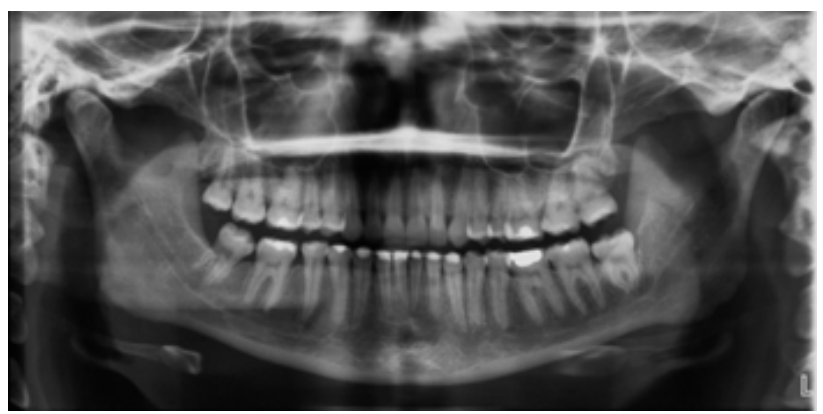

Figura 13 - Radiografia panorâmica final.

Na telerradiografia final observa-se a melhora no perfil e selamento labial, e normalidade das estruturas adjacentes (Figura 14).

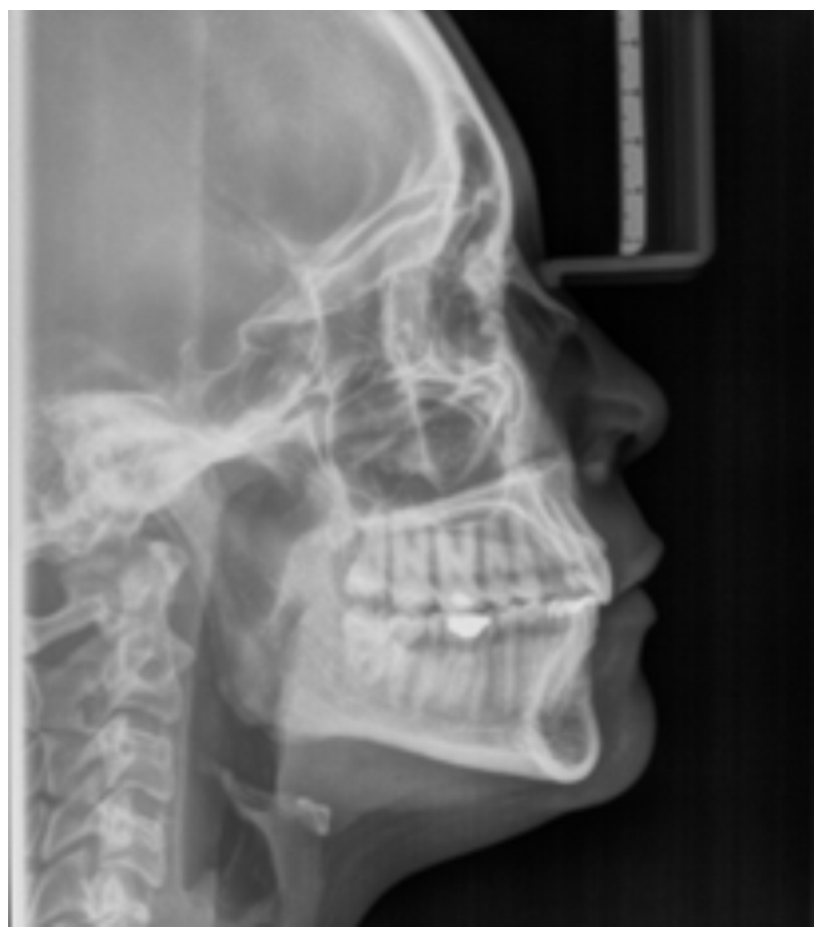

Figura 14 - Telerradiografia final.

Os resultados podem ser vistos clinicamente, porém para confirmar a melhora do posicionamento dentário, utilizou-se a sobreposição das telerradiografias iniciais e finais, sendo as comparações realizadas pelo programa Dolphin ${ }^{\circledR}$. Como o tratamento era ortodôntico e não ortopédico ou cirúrgico, as estruturas esqueléticas e padrão facial não sofreram alterações.

As maiores e mais significantes alterações ocorreram no posicionamento dentário. Houve lingualização e retrusão dos incisivos superiores e inferiores, o que promoveu a melhora do perfil, com aumento significante 
no ângulo nasolabial. Tais alterações ocorreram como efeito colateral da utilização de elásticos em cadeia para o fechamento dos diastemas dentários. Os molares superiores não sofreram alterações significativas. Os molares inferiores sofreram mesialização de $8.7 \mathrm{~mm}$, nota-se que houve movimento de corpo do dente (Figura 15).

\begin{tabular}{|c|c|c|}
\hline & Inicial & Final \\
\hline \multicolumn{3}{|c|}{ Maxila e Base do Crấnio } \\
\hline SNA $\left({ }^{\circ}\right)$ & 80.2 & 80.4 \\
\hline $\mathrm{Co}-\mathrm{A}(\mathrm{mm})$ & 79.8 & 80.3 \\
\hline \multicolumn{3}{|c|}{ Mandíbula e Base do Crânio } \\
\hline SNB $\left({ }^{\circ}\right)$ & 77.4 & 76.9 \\
\hline Co-Gn(mm) & 107.3 & 107.3 \\
\hline \multicolumn{3}{|c|}{ Relaçåo Maxilomandibular } \\
\hline ANB $\left({ }^{\circ}\right)$ & 2.8 & 3.4 \\
\hline Wits (mm) & -4.7 & -0.4 \\
\hline \multicolumn{3}{|c|}{ Componente Vertical } \\
\hline FMA $\left(^{\circ}\right)$ & 26.6 & 25.9 \\
\hline $\mathrm{SN}-\mathrm{GoG} n\left(^{\circ}\right)$ & 32.4 & 30.9 \\
\hline $\mathrm{SN} . \mathrm{OCl}\left({ }^{\circ}\right)$ & 21.7 & 17.9 \\
\hline AFAl $(\mathrm{mm})$ & 65.4 & 64.6 \\
\hline \multicolumn{3}{|l|}{ Dentes Superiores } \\
\hline 1.NA $\left({ }^{\circ}\right)$ & 20.8 & 12.5 \\
\hline $1-\mathrm{PP}(\mathrm{mm})$ & 29.5 & 28.9 \\
\hline $1-\mathrm{NA}(\mathrm{mm})$ & 5.5 & 2.0 \\
\hline Molar - PT Vertical (mm) & 16.9 & 17.3 \\
\hline Molar - SN $\left({ }^{\circ}\right)$ & 76.2 & 72.6 \\
\hline \multicolumn{3}{|l|}{ Dentes Inferiores } \\
\hline 1.NB $\left({ }^{\circ}\right)$ & 27.0 & 22.0 \\
\hline 1-GoMe (mm) & 35.2 & 36.2 \\
\hline $1-\mathrm{NB}(\mathrm{mm})$ & 6.6 & 4.1 \\
\hline Apice do Molar Inferior para Sínfise (mm) & 21.6 & 12.9 \\
\hline $\begin{array}{l}\text { Coroa do Molar Inferior para Sínfise } \\
(\mathrm{mm})\end{array}$ & 22.1 & 13.4 \\
\hline Molar - PP (mm) & 22.2 & 23.3 \\
\hline \multicolumn{3}{|l|}{ Relaçỏes Dentárias } \\
\hline Relação Molar (mm) & 6.2 & -1.1 \\
\hline Sobressaliência $(\mathrm{mm})$ & 2.9 & 2.8 \\
\hline Sobremordida (mm) & 1.5 & 2.6 \\
\hline Angulo Interincisivos $\left({ }^{\circ}\right)$ & 129.5 & 142.0 \\
\hline \multicolumn{3}{|l|}{ Perfil Tegumentar } \\
\hline Lábio Inferior Linha E (mm) & 1.0 & -0.8 \\
\hline Lábio Superior Linha E (mm) & 0.4 & 0.5 \\
\hline Convexidade Facial $\left({ }^{\circ}\right)$ & 9.4 & 9.4 \\
\hline Angulo Nasolabial $\left({ }^{\circ}\right)$ & 95.7 & 103.7 \\
\hline
\end{tabular}

Figura 15 - Valores cefalométricos iniciais e finais.
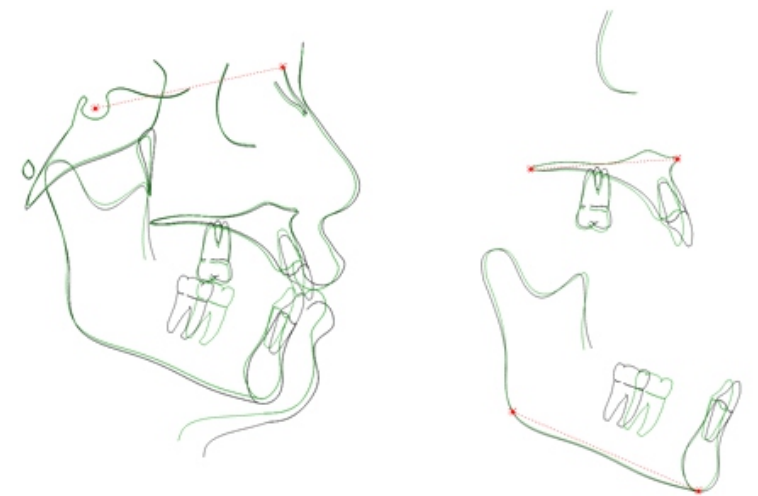

Sobreposição inicial

Sobreposição final

Figura 16 - Sobreposição da telerradiografia final e inicial.

\section{DISCUSSÃO}

$\mathrm{O}$ aumento de pacientes no consultório ortodôntico tendo como queixa principal o espaço causado pela perda de um ou mais dentes, e a possibilidade de tal espaço ser diminuído ou fechado em sua totalidade, tem demonstrado a real necessidade do uso de mini-implantes na clínica ortodôntica, seja para promover uma mesialização, distalização, verticalização ou intrusão .

A mesialização de molares sem a colaboração do paciente, normalmente representa um desafio para o ortodontista, principalmente quando a perda dentária é unilateral, como neste trabalho, pois há uma tendência em desviar a linha média, além de outros efeitos colaterais indesejados ${ }^{7}$. A utilização de mini-implantes facilita a movimentação, a tornando mais simples e eficiente ${ }^{9-11}$.

O movimento de mesialização é um movimento de corpo, e é melhor executado se houver uma verticalização das raízes ${ }^{1}$. Neste trabalho, não foi necessária a verticalização, pois a perda dentária se deu devido a impossibilidade de retratamento do dente 46 , portanto, os dentes 47 e 48 estavam em posição favorável a mesialização, pois ainda não haviam sofrido as movimentações causadas por perdas precoces de dentes adjacentes.

Alguns efeitos colaterais como extrusão e inclinação dos dentes mesializados podem acontecer durante a mecânica de mesialização'. Tais efeitos podem ser minimizados com um melhor controle da mecânica. Neste trabalho, a utilização do cantilever pôde corroborar para que os efeitos indesejáveis não acontecessem, pois, a linha de ação de força se aproxima do centro de resistência do molar, o que faz com que não aconteça a inclinação para mesial, promovendo um movimento de corpo do dente ${ }^{9-13}$.

Quanto ao tempo de tratamento, o ortodontista deve sempre considerá-lo, visto que em média, com condições favoráveis, um tratamento ortodôntico com mesialização de molares aumenta em 1 (um) ano o tempo de tratamento. Na movimentação de segundos ou terceiros molares a taxa de movimentação é de $0.5 \mathrm{~mm} / \mathrm{mês}$, correspondendo à taxa linear da reabsorção osteoclástica ${ }^{14-16}$. Neste caso, além da mesialização a paciente necessitava de tratamento ortodôntico, portanto o tratamento não foi realizado apenas pela necessidade do fechamento do espaço do dente perdido sendo que tal mecânica não comprometeu o tempo de tratamento.

A mesialização neste caso foi de extrema importância, visto que, permitiu que a paciente não necessitasse de reabilitação protética com implantes ao final do tratamento. 


\section{CONCLUSÃO}

Devido à praticidade da técnica de instalação dos miniimplantes, e da independência da colaboração do paciente, conclui-se que a mesialização de molares por meio de miniimplantes é um recurso extremamente útil na clínica ortodôntica beneficiando o paciente, pois diminui-se a necessidade de reabilitação protética.

\section{REFERÊNCIAS}

1. Janson M, Silva F. Mesialização de molares com ancoragem em mini-implantes. R Dental Press Ortodon Ortop Facial. 2008;13(5):88-94.

2. Villela H, Villela P, Bezerra F, Labiossière Júnior MA, Soares AP. Utilização de mini-implantes para ancoragem ortodôntica direta. Innov J. 2005;1:11-8.

3. Egolf RJ, Begole EA, Upshaw HS. Factors associated with orthodontic patient compliance with intraoral elastic and headgear wear. Am J Orthod Dentofac Orthop. 1990;97(4):336-48.

4. Reynders R, Ronchi L, Bipat S. Mini-implants in orthodontics: a systematic review of the literature. Am J Orthod Dentofacial Orthop. 2009;135(5):564.e1-19; discussion 464-5.

5. Gray JB, Smith R. Transitional implants for orthodontic anchorage. J Clin Orthod. 2000;34(11):659-66.

6. Celenza F. Implant-enhanced tooth movement: indirect absolute anchorage. Int J Periodontics Restorative Dent. 2003;23(6):533-41.

7. Gollner N, Winkler J, Gollner P, Gkantidis N. Effect of mandibular first molar mesialization on alveolar bone height: a split mouth study. Prog Orthod. 2019;20:22.

8. Wilmes B, Vasudavan S, Drescher D. Maxillary molar mesialization with the use of palatal mini-implants for direct anchorage in an adolescent patient. Am J Orthod Dentofacial Orthop. 2019;155(5):725-32.

9. Kyung SH, Choi JH, Park YC. Miniscrew anchorage used to protract lower second molars into first molar extraction sites. J Clin Orthod. 2003;37(10):575-9.

10. Kyung SH, Hong SG, Park YC. Distalization of maxillary molars with a midpalatal miniscrew. J Clin Orthod. 2003;37(1):22-6.

11. Poggio PM, Incorvati C, Velo S, Carano A. "Safe zones": a guide for miniscrew positioning in the maxillary and mandibular arch. Angle Orthod. 2006;76(2):191-7.

12. Kyung HM, Park HS, Bae SM, Sung JH, Kim IB. Development of orthodontic micro-implants for intraoral anchorage. J Clin Orthod 2003;37(6):321-8.

13. Kenworthy CR, Larson BE. Incorporating retained deciduous teeth in orthodontic therapy. Am J Orthod Dentofacial Orthop. 2001;119(3):202-10.

14. Goodwin RA, Loyd JE, Des Prez RM. Histoplasmosis in normal hosts. Medicine (Baltimore). 1981;60(4):231-66.

15. Roberts WE, Arbuckle GR, Analoui M. Rate of mesial translation of mandibular molars using implant-anchored mechanics. Angle Orthod. 1996;66(5):331-8.

16. Roberts WE, Marshall KJ, Mozsary PG. Rigid endosseous implant utilized as anchorage to protract molars and close an atrophic extraction site. Angle Orthod 1990;60(2):135-52. 